\title{
REDUCED SUSCEPTIBILITY OF MRSA TO VANCOMYCIN
}

\author{
SUNEEL BHOOSHAN ${ }^{1}$, JYOTI PRASAD ${ }^{2}$, AMITESH DUTTA ${ }^{2}$, VANDANA KE ${ }^{2}$, CHIRANJAY MUKHOPADHYAY²
}

${ }^{1}$ Department of Microbiology AIIMS, Bhopal, India, ${ }^{2}$ Department of Microbiology KMC Manipal, India

Email: vandanake@gmail.com

Received: 23 May 2016 Revised and Accepted: 22 Jul 2016

\section{ABSTRACT}

Objective: This study was conducted to observe the antibiogram, vancomycin MIC (Minimum Inhibitory Concentration), and inducible clindamycin resistance in clinical isolates of MRSA (Methicillin-Resistance Staphylococcus aureus).

Methods: Drug resistance pattern was studied by Kirby-Bauer disc diffusion methods. MIC of vancomycin was determined by agar dilution method.

Results: MRSA was found to be highly resistant to gentamicin (76\%), erythromycin (67.03\%) and ciprofloxacin (65.09\%) while glycopeptides showed uniform susceptibility.

Conclusion: Though there was no drug resistance observed against vancomycin and linezolid, it's wise to use these antibiotics safely as emerging resistance has been reported for these drugs from all over the world.

Keywords: MRSA, Vancomycin Intermediate Staphylococcus aureus, MIC

(C) 2016 The Authors. Published by Innovare Academic Sciences Pvt Ltd. This is an open access article under the CC BY license (http://creativecommons. org/licenses/by/4. 0/) DOI: http://dx.doi.org/10.22159/ijpps.2016v8i9.13023

The emergence of MRSA is the major concern for hospital scenario since these strains are resistant to all $\beta$-lactam antibiotics. However, recent studies on MRSA have shown an increase in MIC of vancomycin, which is the last resort for treating these resistant bugs, leading to failure of vancomycin therapy, especially when MIC value ranges from $1-2 \mu \mathrm{g} / \mathrm{ml}[1,2]$. Therefore, it becomes necessary to look for the vancomycin MIC in all MRSA isolates so that the appropriate treatment may be initiated to obtain treatment success. Herein, we present the laboratory data on the vancomycin MIC among clinical isolates of MRSA and their antibiogram for other non$\beta$-lactam antibiotics.

A total of 465 non-repeat clinical isolates of S. aureus (Staphylococcus aureus) were collected from various clinical specimen including, pus, wound swabs, catheter tip, and blood during January 2010 to January 2011. Identification and susceptibility of the S. aureus were performed by standard tests. Screening of clinical isolates for methicillin resistance was performed by cefoxitin antibiotic disc. Agar plates with gradient vancomycin $(0.25-256 \mu \mathrm{g} / \mathrm{ml})$ were prepared to determine vancomycin MIC. Quality control strains S. aureus ATCC 29213, S aureus ATCC 43300, and Enterococcus faecalis ATCC 51299 were used in our study.

Of the 465 isolates, 282 strains showed resistance to cefoxitin. These strains were reconfirmed for methicillin resistance by using oxacillin agar screen method. Out of 282 cefoxitin resistant strains, 7 strains showed susceptibility in oxacillin screen agar. Therefore 275/465
(59.1\%) clinical strains [205 pus (74.54\%), 40 blood (14.55\%), 7 sputum $(2.55 \%), 2$ throat $(0.73 \%), 21$ catheter-related $(7.64 \%)$ were confirmed as MRSA. The resistance patterns of MRSA isolates to various antimicrobial agents are shown in table 1 . Among all MRSA isolates 32 strains (11.63\%) showed inducible clindamycin resistance (D-Test Positive). Out of which 26 were from pus samples, 4 from blood, and 2 from the catheter. In the present study, the numbers of MRSA isolates were drastically high in pus (wound/aspirate) infections; this might be due to the frequent dressing changes often necessitate a dressing tamper via multiple healthcare workers plus the inherent immune-suppression of the wound patients might lead to MRSA colonization.

The vancomycin MIC for 275 MRSA strains by agar dilution is shown in fig. 1.

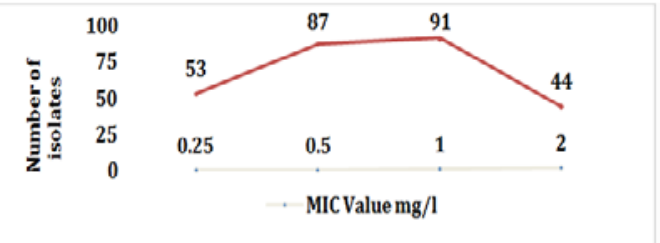

Fig. 1: Distribution of vancomycin MIC among MRSA isolates

Table 1: Resistance profile of MRSA strains against antibiotics

\begin{tabular}{llllll}
\hline S. No. & Antibiotics & Sensitive & & Resistance & Percentage of isolates \\
\cline { 3 - 6 } & & Number of isolates & Percentage of isolates & Number of isolates & 00 \\
\hline 1 & Vancomycin & 275 & 100 & 00 & $76 \%$ \\
2 & Gentamicin & 66 & $24 \%$ & 209 & $65.09 \%$ \\
3 & Ciprofloxacin & 96 & $34.90 \%$ & 179 & $37.45 \%$ \\
4 & Tetracycline & 172 & $62.54 \%$ & 103 & $67.03 \%$ \\
5 & Erythromycin & 94 & $34.18 \%$ & 181 & $35.27 \%$ \\
6 & Clindamycin & 178 & $64.73 \%$ & 97 & $44.00 \%$ \\
7 & Amikacin & 154 & $56.00 \%$ & 121 & $59.27 \%$ \\
8 & Cotrimoxazole & 112 & $43.63 \%$ & 163 & $38.19 \%$ \\
9 & Chloramphenicol & 170 & $61.81 \%$ & 105 & $07.27 \%$ \\
10 & Rifampicin & 255 & $92.72 \%$ & 20 & 00 \\
11 & Linezolid & 275 & 100 & 00 & \\
\hline
\end{tabular}


When these MIC values of were vancomycin arranged according to clinical specimens, we found a lot of variability accordingly (fig. 2).

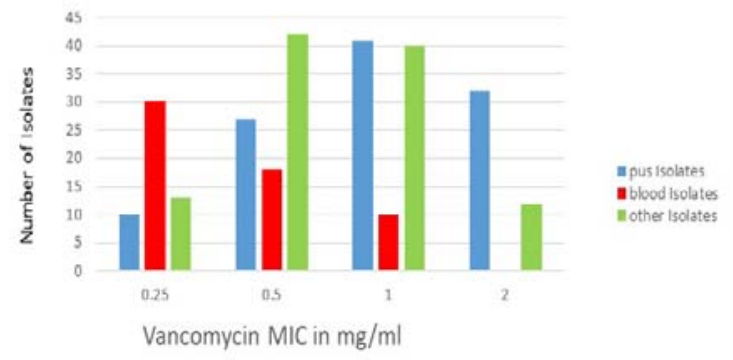

Fig. 2: Correlation between the source of MRSA strains and MIC of vancomycin

A study on the spectrum of antimicrobial resistance among MRSA, gentamycin, erythromycin and ciprofloxacin resistance was as high $63.20 \%, 60 \%$, and $46 \%$ [3]. In contrast, we have $76 \%$ strains resistant to gentamycin, $67.03 \%$ to erythromycin and $65.09 \%$ strains resistant to ciprofloxacin. This might be due to the fact that at the present time these agents are tremendously used in the treatment of general infections. However, a study does not correlate with our findings in the case of gentamycin only $8 \%$ strains resistance to gentamycin as against $76 \%$ in our study [4]. Overall, $11.63 \%$ isolates showed erythromycin inducible clindamycin resistance (D-Test Positive) which is very low in comparison to shown in the study [5]. In this study, we observed $12.69 \%$ inducible clindamycin resistance in plus isolates, $10 \%$ in blood and $09.25 \%$ in isolated from the catheter. The observation of drug resistance in MRSA is leading towards the use of the last resorts of antibiotics such as vancomycin by clinicians, which can be avoided if alternate antibiotic (erythromycin, clindamycin) which has good efficacy and tissue penetration is used in the treatment. This greatly necessitates the need to look for inducible clindamycin resistance. All the isolates of MRSA were sensitive to vancomycin in contrast to recent reports of $S$. aureus isolates with reduced susceptibility to vancomycin. Several studies have also reported similar results from Kolkata and outside India [6-10]. We found a significant number of our isolates belonged to a high MIC group which is alarming. Limitation of the study is drug resistance mechanism to vancomycin in MRSA could be determined by molecular assays.

There is a requirement to identify MRSA quickly to commence effective antimicrobial therapy so that the mortality, complication and treatment cost can be reduced. The MRSA could be prevented by identifying and screening MRSA carriers inside high-risk wards.
We need to encourage and facilitate adherence to recommended prevention and control guidelines, conduct active surveillance to detect the emergence of these organisms, and ensure vigorous antibiotic stewardship by health care providers

\section{CONFLICT OF INTERESTS}

\section{Declared none}

\section{REFERENCES}

1. Fred C Tenover, James W Biddle, Michael V Lancaster. Increasing resistance to vancomycin and other glycopeptides in staphylococcus aureus. Emerging Infect Dis 2001;7:327-32.

2. Hiramatsu K, Hanaki H, Ino T, Yabuta K, Oguri T, Tenover FC Methicillin-resistant Staphylococcus aureus clinical strain with reduced vancomycin susceptibility. J Antimicrob Chemother 1997;40:135-46.

3. Rajaduraipandi K, Mani KR, Panneerselvam K, Mani M, Bhaskar $M$, Manikandan P. Prevalence and antimicrobial susceptibility pattern of methicillin resistant staphylococcus aureus: a multicentre study. Indian J Med Microbiol 2006;24:34-8.

4. Pulimood TB, Lalitha MK, Jesudason MV, Pandian R, Selwyn J, John TJ. The spectrum of antimicrobial resistance among methicillin-resistant staphylococcus aureus (MRSA) in a tertiary care centre in India. Indian J Med Res 1996;103:212-5.

5. Deotale V, Mendiratta DK, Raut U, Narang P. Inducible clindamycin resistance in Staphylococcus aureus isolated from clinical samples. Indian J Med Microbiol 2010;28:124-6.

6. Assadullah S, Kakru DK, Thoker MA, Bhat FA, Hussain N, Shah A. Emergence of low-level vancomycin resistance in MRSA Indian J Med Microbiol 2003;21:196-8.

7. Menezes GA, Harish BN, Sujatha S, Vinothini K, Parija SC Emergence of vancomycin-intermediate staphylococcus species in southern India. J Med Microbiol 2008;57:911-2.

8. Bhateja P, Mathur T, Pandya M, Fatma T, Rattan A. Detection of vancomycin resistance staphylococcus aureus: a comparative study of three different phenotypic screening methods. Indian J Med Microbiol 2005;23:52-5.

9. Veer P, Chande C, Chavan S, Wabale V, Chopdekar K, Bade J, et al. Increasing levels of minimum inhibitory concentration vancomycin in methicillin-resistant Staphylococcus aureus alarming bell for vancomycin abusers. Indian J Med Microbiol 2010;28:413-4.

10. Dhawan B, Gadepalli R, Rao C, Kapil A, Sreenivas V. Decreased susceptibility to vancomycin in meticillin-resistant Staphylococcus aureus: a 5 y study in an Indian tertiary hospital. J Med Microbiol 2010;59:375-6.

\section{How to cite this article}

- Suneel Bhooshan, Jyoti Prasad, Amitesh Dutta, Vandana Ke, Chiranjay Mukhopadhyay. Reduced susceptibility of mrsa to vancomycin. Int J Pharm Pharm Sci 2016;8(9):321-322. 